\title{
Moyenne de localisation fréquentielle des paquets d'ondelettes
}

Ai Hua Fan

Résumé. En utilisant le théorème de Ruelle d'opérateur de transfert, nous démontrons que la moyenne $2^{-k} \sum_{n=0}^{2^{k}-1}\left\|\hat{w}_{n}\right\|_{L^{1}}$ de la localisation fréquentielle pour les paquets d'ondelettes admet un équivalent de la forme $c \rho^{k}(c>0,1<\rho<\sqrt{2})$. Cela améliore une inégalité antérieurement obtenue par Coifman, Meyer et Wickerhauser. Des estimations numériques de $\rho$ sont obtenues pour des filtres de Daubechies.

\section{Enoncé.}

On considère un couple de filtres miroirs conjugués en quadrature $\left(m_{0}, m_{1}\right)$, c'est-à-dire deux fonctions satisfaisant aux conditions suivantes:

C1) $m_{0}$ et $m_{1}$ sont d'une classe lipschitzienne, $2 \pi$-périodiques;

C2) $\left|m_{0}(\xi)\right|^{2}+\left|m_{0}(\xi+\pi)\right|^{2}=1, m_{1}(\xi)=e^{-i \xi} \overline{m_{0}(\xi+\pi)}$;

C3) $m_{0}(0)=1, m_{0}(\xi) \neq 0$ sur un compact $K$ tel que

$$
\bigcup_{j \in \mathbb{Z}}(K+\pi j)=\mathbb{R}
$$


Le produit suivant est alors bien défini

$$
\hat{\varphi}(\xi)=\prod_{j=1}^{\infty} m_{0}\left(\frac{\xi}{2^{j}}\right)
$$

C'est la transformée de Fourier d'une fonction d'échelle $\varphi$. Les paquets d'ondelettes $w_{n}(n \geq 0)$ sont définis par leurs transformées de Fourier comme suit

$$
\hat{w}_{n}(\xi)=\left(\prod_{j=1}^{k} m_{\epsilon_{j}}\left(\frac{\xi}{2^{j}}\right)\right) \varphi\left(\frac{\xi}{2^{k}}\right), \quad \text { si } n=\sum_{j=1}^{k} \varepsilon_{j} 2^{j-1}
$$

Ainsi on a $w_{0}=\varphi ; w_{1}=\psi$, l'ondelette associée au couple $\left(m_{0}, m_{1}\right)$ de filtres miroirs en quadrature [M1].

Pour décrire la localisation fréquentielle de $w_{n}$, on fait appel à la variance de $\hat{w}_{n}$ définie par

$$
\sigma_{n}=\inf _{\omega \in \mathbb{R}} \int_{\mathbb{R}}|\xi-\omega|^{2}\left|\hat{w}_{n}(\xi)\right|^{2} d \xi
$$

L'estimation de $\sigma_{n}$ s'avère un peu délicate. Cependant on obtient facilement la minoration suivante pour $\sigma_{n}$

$$
\left\|\hat{w}_{n}\right\|_{L^{1}(\mathbb{R})} \leq C\left(1+\sigma_{n}\right)^{1 / 2}
$$

où $C$ est une constante indépendante de $n$. Il est prouvé dans [CMW] qu'il existe une constante $r>1$ telle que pour tout $k \geq 1$ on ait

$$
\frac{1}{2^{k}} \sum_{n=0}^{2^{k}-1}\left\|\hat{w}_{n}\right\|_{L^{1}(\mathbb{R})} \geq 2 \pi r^{k}
$$

Nous nous proposons de démontrer dans cette note le théorème suivant.

Théorème. Soit $\left(m_{0}, m_{1}\right)$ un couple de filtres miroirs conjugués en quadrature. Supposons que

$$
\Phi(\xi)=\sum_{j \in \mathbb{Z}}|\hat{\varphi}(\xi+2 \pi j)|
$$


est continue. Alors il existe deux constantes $1<\rho<\sqrt{2}$ et $c>0$ telles qu'on ait l'équivalence

$$
\frac{1}{2^{k}} \sum_{n=0}^{2^{k}-1}\left\|\hat{w}_{n}\right\|_{L^{1}(\mathbb{R})} \sim c \rho^{k} .
$$

Les paquets d'ondelettes ont été introduits en traitement et en compression du signal par Coifman, Meyer et Wickerhauser [CMW]. L'idée remonte à l'analyse en temps-fréquence de signal, qui consiste à décomposer les signaux compliqués en signaux élémentaires caractérisés par leurs localisations en temps et en fréquence. On dit q'un signal $f_{R}(t)$ est localisé dans un rectangle $R=\left[t_{0}-h, t_{0}+h\right] \times\left[\omega_{0}-\pi / h, \omega_{0}+\pi / h\right]$ dans le plan temps-fréquence si

$$
\begin{gathered}
\int_{-\infty}^{+\infty}\left(t-t_{0}\right)^{2}\left|f_{R}(t)\right|^{2} d t \leq K^{2} h^{2} \\
\int_{-\infty}^{+\infty}\left(\omega-\omega_{0}\right)^{2}\left|\hat{f}_{R}(\omega)\right|^{2} d \omega \leq \frac{2 \pi K^{2}}{h^{2}} .
\end{gathered}
$$

Un tel signal, dit atome temps-fréquence, est considéré comme élémentaire. On souhaite aussi que ces atomes soient orthogonaux. L'exemple le plus célèbre d'atomes temps-fréquence est celui de Gabor qui réalise la meilleure constante $K$. Mais les ondelettes de Gabor ne sont pas orthogonales, même pas presque orthogonales [S1]. Dans la littérature, il y avait aussi les ondelettes de Malvar. Celles-ci sont orthogonales par construction. Mais la localisation n'est pas satisfaite. D'ailleurs, les ondelettes de Malvar ne sont pas obtenues par translation, changement d'échelles et modulation d'une fonction fixée une fois pour toutes. C'est pour quoi on a introduit les paquets d'ondelettes [M2]. Elles sont orthogonales et obtenues à partir d'une seule fonction par translation, changement d'échelle et modulation. Mais comme le montrent l'inégalité de Coifman-Meyer-Wickerhauser et le théorème énoncé ci dessus, certain paquets d'ondelettes ont une mauvaise localisation temps-fréquence. Signalons que des estimations de la localisation pour un paquet individuel sont obtenues dans [S1]. 


\section{Preuve.}

La preuve du théorème se décompose en une série de lemmes. Pour simplifier, notons

$$
M_{\varepsilon}=\left|m_{\varepsilon}\right|, \quad \varepsilon=0,1, \quad S=M_{0}+M_{1} .
$$

Lemme 1. Soient $q>0$ un réel, $h(\xi) \geq 0$ une fonction homogène d'ordre $\tau$. Si $n=\sum_{j=1}^{k} \varepsilon_{j} 2^{j-1}$, on a

$$
\left\|h \hat{w}_{n}^{q}\right\|_{L^{1}(\mathbb{R})}=2^{k(1+\tau)} \int_{0}^{2 \pi}\left(\prod_{j=1}^{k} M_{\varepsilon_{j}}\left(2^{j-1} \xi\right)\right)^{q} \Phi_{h, q}(\xi) d \xi,
$$

où

$$
\Phi_{h, q}(\xi)=\sum_{j \in \mathbb{Z}} h(\xi+2 \pi j)|\hat{\varphi}(\xi+2 \pi j)|^{q} .
$$

Preuve. Par le théorème de convergence monotone, on a

$$
\left\|h \hat{w}_{n}^{q}\right\|_{L^{1}(\mathbb{R})}=\lim _{J \rightarrow \infty}\left\|h(\xi)\left(\prod_{j=1}^{J} M_{\varepsilon_{j}}\left(\frac{\xi}{2^{j}}\right)\right)^{q} \hat{\varphi}^{q}\left(\frac{\xi}{2^{J}}\right)\right\|_{L^{1}\left(\left[-2 \pi 2^{J}, 2 \pi 2^{J}\right]\right)} .
$$

Or, si $J \geq k$, l'intégrale à droite est égale à

$$
\begin{aligned}
& \int_{-2 \pi 2^{J}}^{2 \pi 2^{J}} h(\xi)\left(\prod_{j=1}^{k} M_{\varepsilon_{j}}\left(\frac{\xi}{2^{j}}\right)\right)^{q}\left|\hat{\varphi}\left(\frac{\xi}{2^{k}}\right)\right|^{q} d \xi \\
&=2^{k(1+\tau)} \int_{-2 \pi 2^{J-k}}^{2 \pi 2^{J-k}}\left(\prod_{j=1}^{k} M_{\varepsilon_{j}}\left(2^{j-1} y\right)\right)^{q} h(y)|\hat{\varphi}(y)|^{q} d y \\
&=2^{k(1+\tau)} \int_{0}^{2 \pi}\left(\prod_{j=1}^{k} M_{\varepsilon_{j}}\left(2^{j-1} y\right)\right)^{q} \\
& \cdot \sum_{j=-2^{J-k}}^{2^{j-k}-1} h(y+2 \pi j)|\hat{\varphi}(y+2 \pi j)|^{q} d y .
\end{aligned}
$$

Pour obtenir la première égalité on a fait le changement de variable $\xi=2^{k} y$ et utilisé l'homogénéité de $h$. Pour obtenir la seconde on a 
utilisé la périodicité de $M_{\varepsilon}\left(2^{j} y\right)$. Afin de terminer la preuve il suffit d'appliquer encore une fois le théorème de convergence monotone.

Le Lemme 1 est énoncé sous une forme un peu plus générale que ce dont on a besoin. En fait, le choix $q=1$ et $h=1$ sera suffisant pour déduire le lemme suivant.

Lemme 2. Pour tout $k \geq 1$, on a

$$
\frac{1}{2^{k}} \sum_{n=0}^{2^{k}-1}\left\|\hat{w}_{n}\right\|_{L^{1}(\mathbb{R})}=\int_{0}^{2 \pi} \prod_{j=1}^{k} S\left(2^{j-1} \xi\right) \Phi(\xi) d \xi .
$$

PREUVE. Il suffit de remarquer que

$$
\sum_{\varepsilon_{1}, \ldots, \varepsilon_{k}} \prod_{j=1}^{k} M_{\varepsilon_{j}}\left(2^{j-1} \xi\right)=\prod_{j=1}^{k} S\left(2^{j-1} \xi\right) \text {. }
$$

Le Lemme 3 va établir une relation entre l'intégrale à droite dans le Lemme 2 et un opérateur de transfert, qui est défini par

$$
L_{S} f(\xi)=S\left(\frac{\xi}{2}\right) f\left(\frac{\xi}{2}\right)+S\left(\frac{\xi}{2}+\pi\right) f\left(\frac{\xi}{2}+\pi\right) .
$$

Lemme 3. Pour toute fonction $\Phi$ définie sur $[0,2 \pi]$, on a

$$
2^{k} \int_{0}^{2 \pi} \prod_{j=1}^{k} S\left(2^{j-1} \xi\right) \Phi(\xi) d \xi=\int_{0}^{2 \pi} L_{S}^{k} \Phi(\xi) d \xi
$$

Preuve. On prouve l'égalité pour $n=1$. Le cas général se demontre par récurrence.

$$
\begin{aligned}
\int_{0}^{2 \pi} L_{S} \Phi(\xi) d \xi & =\int_{0}^{2 \pi} S\left(\frac{\xi}{2}\right) \Phi\left(\frac{\xi}{2}\right) d \xi+\int_{0}^{2 \pi} S\left(\frac{\xi}{2}+\pi\right) \Phi\left(\frac{\xi}{2}+\pi\right) d \xi \\
& =2 \int_{0}^{\pi} S(y) \Phi(y) d y+2 \int_{\pi}^{2 \pi} S(z) \Phi(z) d z \\
& =2 \int_{0}^{2 \pi} S(x) \Phi(x) d x
\end{aligned}
$$


Pour la seconde égalité, on a fait les changements de variables $\xi / 2=y$ et $\xi / 2+\pi=z$.

Preuve du ThÉorème. L'opérateur de transfert $L_{S}: C(\mathbb{T}) \rightarrow C(\mathbb{T})$ est bien défini et borné, où $\mathbb{T}=\mathbb{R} /(2 \pi \mathbb{Z})$. On note $\lambda$ son rayon spectral. Comme $S$ est strictement positive et lipschitzienne, d'après le théorème d'opérateur de transfert dû à Ruelle $[\mathrm{R}]$ (voir aussi $[\mathrm{B}],[\mathrm{F}],[\mathrm{W}]$ ), il existe une mesure de probabilité $\nu$ et une fonction continue $\ell>0$ telles que, pour toute fonction continue $f, \lambda^{-k} L_{S}^{k} f$ converge uniformément vers $\langle f, \nu\rangle \ell$ où $\langle f, \nu\rangle$ désigne l'intégrale de $f$ par rapport à $\nu$. De plus, on sait que $\nu$ est diffuse, singulière et de support $[0,2 \pi]$. Par conséquent,

$$
\lambda^{-k} \int_{0}^{2 \pi} L_{S}^{k} \Phi(\xi) d \xi \longrightarrow\langle\Phi, \nu\rangle\langle\ell, \nu\rangle=c>0
$$

d'où l'équivalence dans le théorème avec $\rho=\lambda / 2$. Il reste à expliquer que $1<\rho<\sqrt{2}$, ou bien $\log 2<\log \lambda<3 \log 2 / 2$. Or, $\log \lambda$ est la pression de $\log S$ sous la transformation $T(x)=2 x(\bmod 2 \pi)$. Rappelons le principe variationnel $[\mathrm{B}],[\mathrm{R}],[\mathrm{W}]$

$$
\log \lambda=\sup \left(H(\mu)+\int_{0}^{2 \pi} \log S(\xi) d \mu(\xi)\right)
$$

où le sup est pris sur l'ensemble des mesures $T$-invariantes; il est atteint uniquement en $\mu_{S}=\ell \nu, H(\mu)$ désignant l'entropie de $\mu$. Alors, comme $S(\xi) \leq \sqrt{2}$, on a

$$
\log \lambda=h_{\mu_{S}}+\int_{0}^{2 \pi} \log S(\xi) d \mu_{S}(\xi) \leq h_{\mu_{S}}+\frac{1}{2} \log 2<\log 2+\frac{1}{2} \log 2 .
$$

Pour la dernière inégalité on a utilisé le fait que l'entropie maximale de $T$ est égale à $\log 2$ et que la mesure de Lebesgue est l'unique mesure d'entropie maximale. Notons $\mu_{0}$ la mesure de Lebesgue. Comme $S(\xi) \geq$ 1 , on a

$$
\log \lambda>h_{\mu_{0}}+\int_{0}^{2 \pi} \log S(\xi) d \mu_{0}(\xi) \geq h_{\mu_{0}}=\log 2
$$




\section{Filtres de Daubechies.}

On considère les filtres de Daubechies de la forme suivante [D1], [D2], qui dépendent d'un paramètre entier $N \geq 1$ [D1], [D2]. Au lieu d'écrire $m_{0}$, on écrit $m_{0, N}$, qui est défini par

$$
m_{0, N}(\xi)=\left(\frac{1}{2}\left(1+e^{i \xi}\right)\right)^{N} Q_{N}(\xi)
$$

où $Q_{N}$ est un polynôme tel que

$$
\left|Q_{N}(\xi)\right|^{2}=\sum_{k=0}^{N-1}\left(\begin{array}{c}
N-1+k \\
k
\end{array}\right) \sin ^{2 k}\left(\frac{\xi}{2}\right) .
$$

Pour $N \geq 1$, notons $\rho_{N}$ le $\rho$ correspondant dans le théorème. Numériquement, on a

$$
\begin{array}{ll}
\rho_{2}=1,20947, & \rho_{3}=1,17270, \\
\rho_{4}=1,14924, & \rho_{5}=1,13283, \\
\rho_{6}=1,12062, & \rho_{7}=1,11114, \\
\rho_{8}=1,10354, & \rho_{9}=1,09730 .
\end{array}
$$

La méthode de calcul numérique se trouve dans [FL]. Il s'agit d'approcher la fonction $S$ par une fonction en escalier dont le rayon spectral de l'opérateur associé est celui d'une matrice.

\section{References.}

[B] Bowen, R., Equilibrium states and the ergodic theory of Anosov diffeomorphisms. Lecture Notes in Math. 470. Springer, 1975.

[CMW] Coifman, R. R., Meyer, Y., Wickerhauser, V. M., Size properties of wavelets packets. In Wavelets and their applications. Beylkin and al. eds., Jones and Bartlett, 450-470.

[D1] Daubechies, I., Orthonormal bases of compactly supported wavelets. Comm. Pure Appl. Math. 41 (1988), 909-996.

[D2] Daubechies, I., Ten lectures on wavelets. CBMS-NSF Regional Conference Series in Applied Mathematics, 1992.

[F] Fan, A. H., A proof of the Ruelle operator theorem. Reviews in Math. Phys. 7 (1995), 1241-1247. 
[FL] Fan, A. H., Lau, K. S., Asymptotic behavior of multiperiodic functions $G(x)=\prod_{n=1}^{\infty} g\left(x / 2^{n}\right)$. To appear in J. Fourier Anal. Appl.

[M1] Meyer, Y., Ondelettes et opérateurs I. Hermann, 1990.

[M2] Meyer, Y., Ondelettes et applications. Armand Colin, 1992.

[R] Ruelle, D., Thermodynamic formalism: the mathematical structures of classical equilibrium statistical mecanics. Encyclopedia of mathematics and its applications 5. Addison-Wesley (1978).

[S1] Séré, E., Localisation fréquentielle des paquets d'ondelettes. Revista Mat. Iberoamericana 11 (1995), 334-354.

[S2] Séré, E., Thèse, CEREMADE, Université Paris-Dauphine, (1992).

[W] Walters, P., An introduction to ergodic theory. Springer-Verlag, 1982.

Recibido: 8 de octubre de 1.996

Ai Hua Fan

Département de Mathématiques

Université de Cergy Pontoise

95302 Cergy Pontoise, FRANCE

fan@math.pst.u-cergy.fr 